\title{
Gravitational waves from black-hole mergers
}

\author{
By JOHN G. BAKER ${ }^{1}$, WILLIAM D. BOGG ${ }^{2}$, \\ JOAN M. CENTRELLA ${ }^{1}$, BERNARD J. KELL Y ${ }^{1}$, \\ SEAN T. McWILLIAMS ${ }^{2}$, AND JAMES R. van METER \\ ${ }^{1}$ Gravitational Astrophysics Laboratory, NASA Goddard Space Flight Center, 8800 Greenbelt \\ Rd., Greenbelt, MD 20771, USA \\ ${ }^{2}$ University of Maryland, Department of Physics, College Park, MD 20742, USA \\ ${ }^{3}$ Center for Space Science \& Technology, University of Maryland Baltimore County, Physics \\ Department, 1000 Hilltop Circle, Baltimore, MD 21250, USA
}

\begin{abstract}
Coalescing black-hole binaries are expected to be the strongest sources of gravitational waves for ground-based interferometers as well as the space-based interferometer LISA. Recent progress in numerical relativity now makes it possible to calculate the waveforms from the strong-field dynamical merger and is revolutionizing our understanding of these systems. We review these dramatic developments, emphasizing applications to issues in gravitational wave observations. These new capabilities also make possible accurate calculations of the recoil or kick imparted to the final remnant black hole when the merging components have unequal masses, or unequal or unaligned spins. We highlight recent work in this area, focusing on results of interest to astrophysics.
\end{abstract}

\section{Introduction}

Gravitational wave astronomy will open a new observational window on the universe. Since large masses concentrated in small volumes and moving at high velocities generate the strongest and therefore most readily detectable waves, the final coalescence of blackhole binaries is expected to be one of the strongest sources. The opening of the full electromagnetic spectrum to astronomical observation during the last century greatly expanded our understanding of the cosmos. In this new century, observations across the gravitational wave spectrum will provide a wealth of new knowledge, including accurate measurements of binary black-hole masses and spins.

The high frequency part of the gravitational wave spectrum, $\sim 10 \mathrm{~Hz} \lesssim f \lesssim 10^{3} \mathrm{~Hz}$, is being opened today through the pioneering efforts of first-generation ground-based interferometers such as LIGO, currently operating at design sensitivity. Such instruments can detect gravitational waves from coalescing stellar-mass $\left(M \lesssim 10^{2} M_{\odot}\right)$ and intermediate-mass $\left(10^{2} M_{\odot} \lesssim M \lesssim 10^{3} M_{\odot}\right)$ black-hole binaries. While detections from this first generation of detectors are likely to be rare, the advanced LIGO (adLIGO) upgrade may detect the coalescence of several stellar-mass and tens of intermediate-mass black-hole binaries per year. Other high-frequency sources include binary neutron star coalescences, supernovae, and rotating neutron stars.

The low frequency gravitational wave window, $3 \times 10^{-5} \mathrm{~Hz} \lesssim f \lesssim 1 \mathrm{~Hz}$, is especially rich in astrophysical sources and will be opened by the space-based LISA detector, currently in the formulation stage. LISA will be sensitive to the coalescence of massive black-hole binaries with total masses in the range $10^{4} M_{\odot} \lesssim M \lesssim 10^{7} M_{\odot}$ to large redshifts $z \gtrsim 10$ at relatively high signal-to-noise ratios (SNRs), and may detect 10 or more such events per year. Using such observations, the black-hole masses, spins and luminosity distances can be determined to very good precision, with errors $<1 \%$ in some cases (Lang \& Hughes 2006). In addition, LISA will detect gravitational waves from the inspiral of compact stars into central massive black holes out to $z \sim 1$, as well as tens of thousands of compact binaries in the Galaxy. 
The actual merger of two comparable-mass black holes that plunge together and form a common event horizon takes place in the strong-field dynamical regime of general relativity. For many years, we were unable to calculate the expected waveforms from these very energetic events due to severe problems with the large scale computer codes needed to simulate the mergers. Recently, however, a series of stunning breakthroughs has occurred in numerical relativity, resulting in stable, robust, and accurate simulations of black-hole mergers as well as applications to astrophysics. In Sec. 2 we review these developments and present examples of the resulting gravitational waveforms. Applications of these signals to issues in gravitational wave observations are discussed in Sec. 3. When the merging black holes have unequal masses, or unequal or unaligned spins, the final remnant black hole suffers a recoil; recent progress in calculating these "kicks" and their applications to astrophysics are presented in Sec. 4. We conclude with a summary in Sec. 5 .

\section{Calculating black-hole binary coalescence}

The final coalescence of a black-hole binary is driven by gravitational wave emission, and proceeds in three stages: an adiabatic inspiral, a dynamical merger, and a final ringdown (Flanagan \& Hughes 1998). During the inspiral, the black holes are well-separated and can be approximated as point particles. The black holes spiral together on quasicircular trajectories, and the resulting gravitational waveforms are chirps, i.e. sinusoids that increase in both frequency and amplitude as the black holes get closer together. The inspiral can be treated analytically using the post-Newtonian (PN) approach, which is an expansion in $v / c$, where $v$ is the characteristic orbital velocity (see Blanchet (2006) for a review of PN results). The inspiral is followed by a dynamical merger in which the black holes plunge together to form a highly distorted single black hole, producing a powerful burst of gravitational radiation. Since the merger stage occurs in the regime of very strong gravity, a full understanding of this process requires numerical relativity simulations of the Einstein equations. After merger, the remnant black hole then settles down, evolving towards a quiescent Kerr state by shedding its nonaxisymmetric modes as gravitational waves. The late part of this ringdown stage can be treated analytically using black-hole perturbation theory, and the resulting gravitational waveforms are superpositions of exponentially damped sinusoids of constant frequency (Leaver 1986; Echeverría 1989).

In numerical relativity, the full set of Einstein's equations are solved on a computer in the dynamical, nonlinear regime. This is typically accomplished by slicing 4-D spacetime into a stack of 3-D spacelike hypersufaces, each labeled by time $t$ (Arnowitt et al. 1962; Misner et al. 1973). The Einstein equations split into two sets. The constraints give a set of relationships that must hold on each slice and in particular constrain the initial data for a black-hole binary simulation. This data is then propagated forward in time using the evolution equations. Four freely-specifiable coordinate, or gauge, conditions give the development of the spatial and temporal coordinates during the evolution.

Simulating the merger of a black-hole binary using numerical relativity has proved to be very challenging. The first attempt to evolve a head-on collision in 2-D axisymmetry dates back to 1964 (Hahn \& Lindquist 1964). In the mid-1970s, the head-on collision of two equal mass, nonspinning black holes was first simulated successfully, along with the extraction of some information about the gravitational radiation (Smarr et al. 1976; Smarr 1977, 1979). In the 1990s, fully 3-D numerical relativity codes were developed and used to evolve grazing collisions of black holes (Brügmann 1999; Brandt et al. 2000; Alcubierre et al. 2001). However, the codes were plagued by a host of instabilities that 
caused them to crash before any significant portion of a black-hole binary orbit could be evolved. For many years, progress was slow and incremental.

Recently, a series of dramatic developments has led to major progress in black-hole binary simulations across a broad front. The first complete orbit of a black-hole binary was achieved in 2004 (Brügmann et al. 2004). This was followed by the first full simulation of a black-hole binary through an orbit, plunge, merger and ringdown in 2005 (Pretorius 2005). In late 2005, the development of new coordinate conditions produced a breakthrough in the ability to carry out accurate and stable long-term evolutions of black-hole binaries (Campanelli et al. 2006a; Baker et al. 2006c; van Meter et al. 2006). These novel but simple "moving puncture" techniques proved highly effective. They were quickly adopted by a broad segment of the numerical relativity community, leading to stunning advances in black-hole binary modeling, starting with evolutions of equal mass, nonspinning black holes and moving quickly to include unequal masses and spins; (see, e.g. , Campanelli et al. (2006b); Baker et al. (2006b); Campanelli et al. $(2006 d)$; Gonzalez et al. (2007b); Baker et al. (2007b); Herrmann et al. (2006, 2007); Campanelli et al. (2007a,b); Koppitz et al. (2007); Gonzalez et al. (2007a); Baker et al. (2007a); Tichy \& Marronetti (2007)).

The most rapid advances in modeling black-hole binary coalescences cover the previously least understood part of the gravitational waveform, i.e. the final few cycles of radiation generated from near the "innermost stable circular orbit" (ISCO) and afterward, which we call the "merger-ringdown". There is already considerable progress toward a full understanding of this important "burst" portion of the waveform, through which the frequency sweeps by a factor of $\sim 3$ up to ringdown and during which the gravitational wave luminosity is $\sim 10^{23} L_{\odot}$, more than the luminosity of the combined starlight in the visible universe.

A particularly significant development was the demonstration of initial-data-independence of merger-ringdown waveforms for equal-mass, nonspinning black holes (Baker et al. 2006b), as summarized in Fig. 1. Results from four runs with successively larger initial separations are shown; the waveforms have been aligned so that the moment of peak radiation amplitude in each simulation occurs at time $t=0$. Here we show the gravitational wave strain from the dominant $(l=2, m=2)$ mode; this represents an observation made on the equatorial plane of the system, where only a single polarization component contributes to the measured strain. The upper panel of Fig. 1 1 shows the full simulation waveforms, while the lower panel focuses on the final merger-ringdown burst. Note that here and elsewhere in this paper, we use geometrical units, $G=c=1$, to measure time, distance and mass in the same units. In particular, one solar mass $M_{\odot}$ is equivalent to $\sim 5 \times 10^{-6} \mathrm{sec}$, or $\sim 1.5 \mathrm{~km} \forall$ In Fig. 11, our timescale is the final mass $m_{f}$, of the post-merger hole; this will be less than the initial total binary mass $M$ because of gravitational radiation. In the shortest run (solid line), the black holes are placed on initial orbits close to the ISCO and undergo a brief plunge followed by a merger and ringdown (Baker et al. 2006c). At the next-largest initial separation (dashed line), the black holes complete $\sim 1.8$ orbits before merging (Baker et al. 2006b). The waveforms from the two runs with successively larger initial separations (dot-dashed and dotted lines, respectively) then lock on to the merger-ringdown part of this shorter (dashed) run. In fact, the waveforms from the three longer simulations show very strong agreement for $t \gtrsim-50 m_{f}$, with differences among these waveforms $\lesssim 1 \%$ in this regime.

Today, there is consensus that the merger of equal-mass, nonspinning black holes pro-

$\dagger$ Since the simulation results scale with the masses of the black holes, they are equally applicable to LISA and ground-based detectors. 


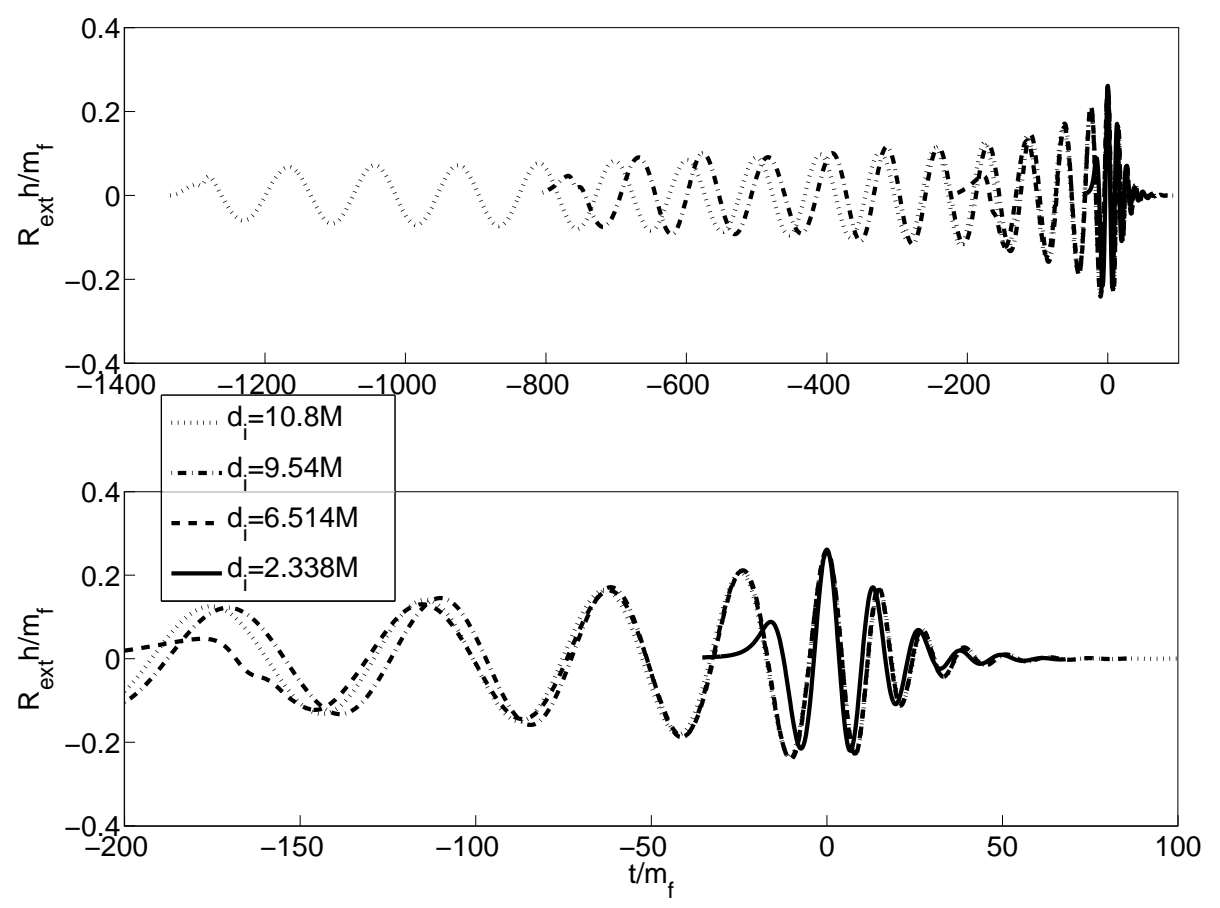

Figure 1 Gravitational waveforms are shown for four simulations of equal mass nonspinning black-hole binaries with successively wider initial separations. Here, $d_{i}$ is the initial coordinate separation of the black-hole centers ("punctures"). All waveforms have been aligned so that the moment of maximum radiation amplitude occurs at $t=0$ (phase is also set to zero at $t=0$ ). The upper panel shows the full extent of all the runs, while the lower panel shows the final merger-ringdown burst.

duces a final Kerr black hole with spin $a / m_{f} \sim 0.7$, and that the amount of energy radiated in the form of gravitational waves, starting with the final few orbits and proceeding through the plunge, merger and ringdown, is $E_{\mathrm{rad}}=M-m_{f} \sim 0.04 M$ (Pretorius 2005; Campanelli et al. 2006a; Baker et al. 2006c; Campanelli et al. 2006b; Baker et al. $2006 \mathrm{~b}$ ); see Centrella (2006) for a review. There is also agreement on the overall simple shape of the waveforms shown in Fig. 1, and detailed comparison of results among numerical relativity groups has already begun (Baker et al. 2006a). Parameter-space exploration of the merger-ringdown burst, using simulations with various mass ratios (Herrmann et al. 2006; Baker et al. 2006d; Gonzalez et al. 2007b) and spins (Campanelli et al. $2006 d . c$ ), is now underway. The next step is to push this frontier to increasingly complex mass-ratio and spin-orientation combinations, and to establish initial-data independence across these parameters.

Simulations starting in the late-inspiral regime and covering more than a few orbits prior to merger are more challenging than shorter merger-ringdown evolutions (Baker et al. $2007 b)$. Such long simulations have stronger requirements for numerical stability and place greater demands on computational resources. In addition, better accuracy is needed to control the accumulation of phase error; this in turn constrains the numerical error 
that can be tolerated in the rate of energy loss through gravitational radiation reaction, which governs the inspiral.

So far, the longest simulations have been carried out for equal-mass, nonspinning blackhole binaries, starting at relatively wide separations. The two longest runs shown in Fig. 1 undergo $\sim 4.2$ (Baker et al. 2006b) and $\sim 7$ orbits (Baker et al. 2007b), respectively, prior to merget and demonstrate progress in long simulations (see also Husa et al. (2007a)). We expect that astrophysical black-hole binaries in the late inspiral regime will follow nearly circular orbits, since any initial eccentricity would have been radiated away early in their evolution. However, in the first long black-hole binary simulations, the eccentricity of the initial orbits was not very well controlled; in particular, the early part of the waveform shown by the dot-dashed line shows evidence of eccentricity which resulted from the initial data specification. The dotted curve shows more recent results, which start with very small eccentricity $\epsilon<0.01$ (see also Pfeiffer et al. (2007); Husa et al. (2007b)). Such long waveforms make it possible to compare the results of numerical relativity simulations of black-hole binaries with post-Newtonian calculations in the late-inspiral regime (Buonanno et al. 2007a; ; Baker et al. 2006e; Hannam et al. 2007), demonstrating remarkable agreement.

\section{Observing Black-Hole Binary mergers}

The final moments of a black-hole binary merger produce the most intense radiation generated through the strongest gravitational dynamics. The resulting waveforms are obviously an important part of the observable gravitational wave signature for blackhole binary events. Before these recent advances in numerical relativity, however, little was confidently known about these signals, so that observational analyses of gravitational wave data could only rely on generic "unmodeled burst" techniques (Abbott et al. 2007) rather than more effective matched-filtering techniques, which require detailed knowledge of the expected signals. Consequently the quality of scientific information which could be expected from merger observations has, to date, necessarily been significantly discounted in planning observational work.

Just how much more we can learn from observing the final stages of black-hole binary mergers with full knowledge of the waveforms, and how best to apply this knowledge in gravitational wave data analysis, are still largely unanswered questions, which the gravitational wave community is only beginning to address. We can get some rough impressions of the observational significance of black-hole binary mergers from this new perspective by considering the SNR of the full waveforms based on matched-filtering analysis of gravitational wave data (Flanagan \& Hughes 1998). Estimating the full waveforms by stitching together the most accurate PN model with a numerical simulation of the last several cycles, we have calculated the SNR for equal-mass nonspinning binaries (Baker et al. $2007 b)$. Fig. 2 shows the contours of SNR as functions of redshift $z$ and total binary mass $M$ for the ground-based adLIGO detector, which will be sensitive in the frequency range $14 \mathrm{~Hz} \lesssim f \lesssim 10^{3} \mathrm{~Hz}$. AdLIGO is a planned upgrade of the initial LIGO detectors that will increase the sensitivity by roughly an order of magnitude across the frequency band. In addition, adLIGO can be tuned to optimize its sensitivity for different sources. To produce Fig. 2 we used the wide-band tuning typically associated with burst sources, due to its greater sensitivity at higher frequencies, where the merger portion from many sources is predicted to occur (Shoemaker 2006). This gives an improved SNR for most

$\dagger$ For these quadrupolar waves, the gravitational wave and orbital frequencies are related by $f=2 f_{\text {orb }}$. 


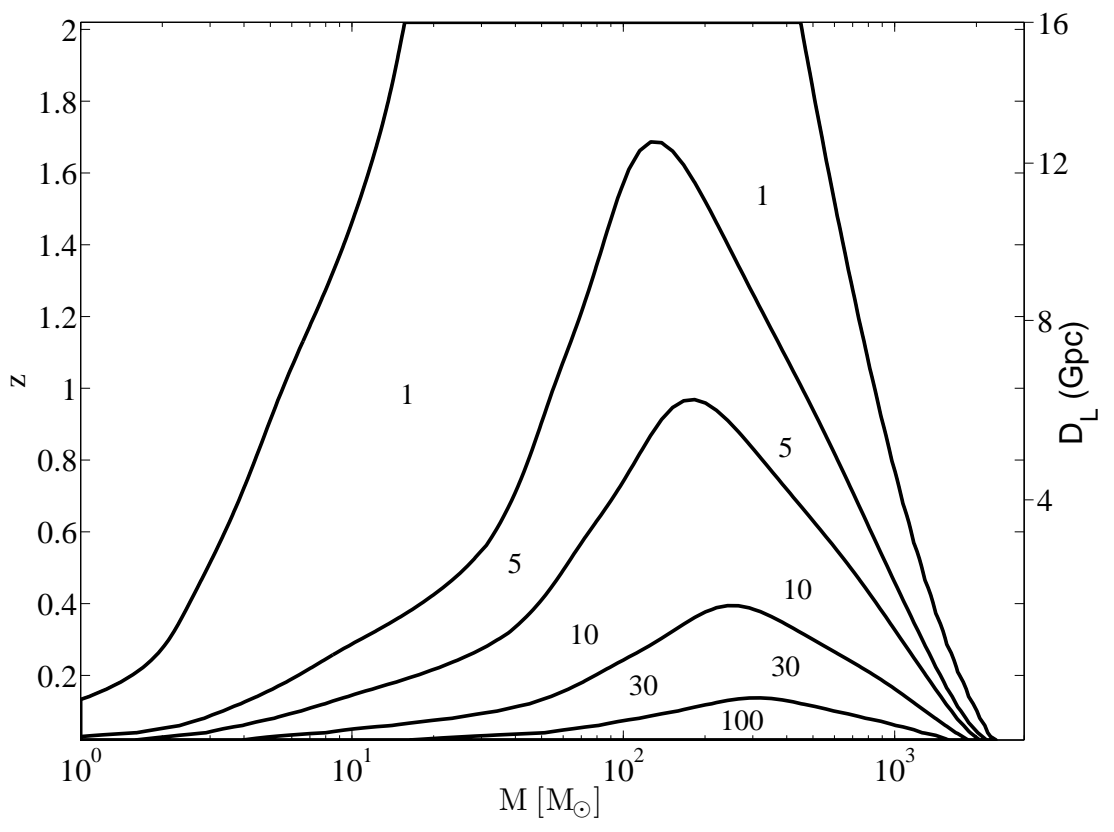

Figure 2 Contours of SNR for observations of black-hole binaries with adLIGO, showing mass, redshift, and luminosity-distance dependence.

black-hole masses compared to tunings that were optimized for only the early inspiral portion of the coalescence. In Fig. 3 we plot contours of SNR for LISA observations, showing that LISA can observe massive black-hole binaries at high SNR throughout the observable universe. LISA is most sensitive to systems with masses in the range $10^{5} M_{\odot} \leqslant M \leqslant 10^{7} M_{\odot}$, coinciding with the mass range in which models of massive black-hole binaries predict coalescence within a Hubble time (Milosavlievic \& Merritt 2003) and event rates for LISA of at least several per year (Sesana et al. 2005).

In Fig. 4 we plot a simulated LISA data stream containing a black-hole binary signal as well as noise from the interferometer and the white dwarf confusion noise caused from the unresolvable number of background white dwarf binaries. This is an example of a typical, non-optimal case in order to demonstrate the relative strength that these signals will generally have. Here we show two $4 \times 10^{5} M_{\odot}$ black holes at redshift $z=10$, inclination $\iota=\pi / 2$, polarization angle $\psi=0$, ecliptic latitude $\beta=0$, and ecliptic longitude $\lambda=\pi$. So far we have restricted our consideration to the example of equalmass nonspinning black-hole binary mergers. More detailed considerations of black-hole binary observations must take into account the full parameter space of these systems, which, from the point of view of general relativity, are described by seven parameters, corresponding to the black-hole mass-ratio $q \equiv m_{1} / m_{2} \leqslant 1$ (recall that the total mass $M$ only scales the waveforms), and the spin vector of each hole (plus two more parameters if eccentric systems are considered). Much progress has already been made in conducting numerical simulations to flesh out the basic features of this parameter space (see, e.g. , Campanelli et al. (2006c, d) ; Herrmann et al. (2006)). First impressions indicate that the waveforms in this parameter space are qualitatively simple, with smooth parameter dependencies. This suggests that our knowledge of the mergers can ultimately be applied in observations with the aid of smooth analytic waveform models, joining PN treatments 


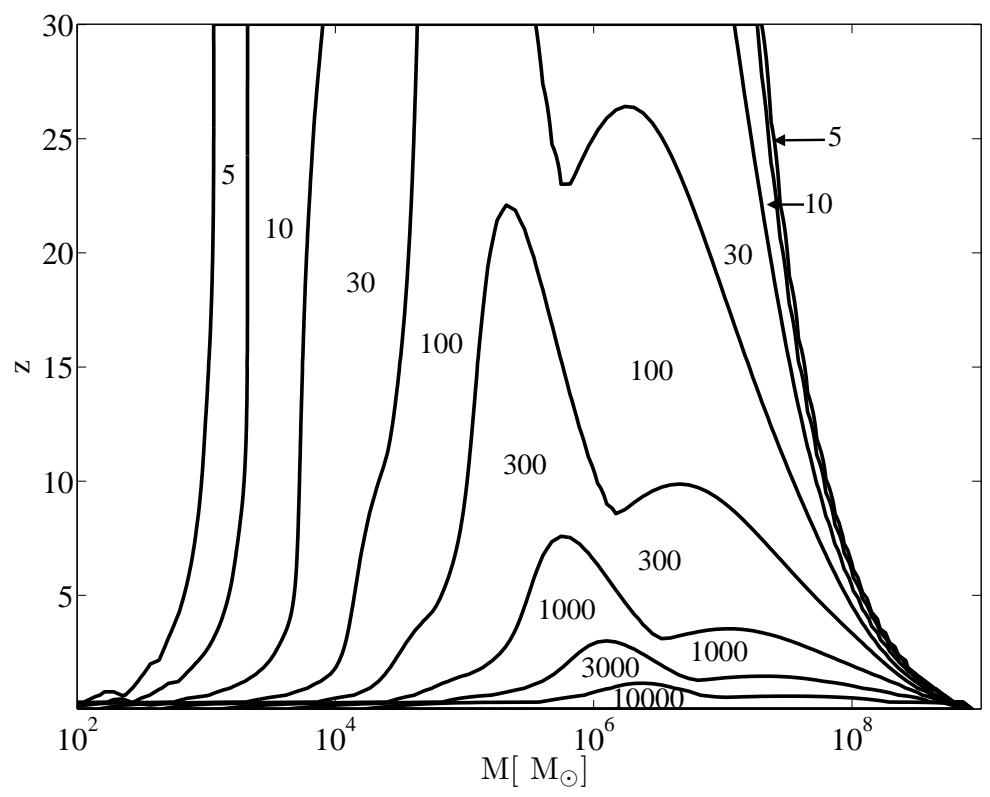

Figure 3 Contours of LISA SNR with mass, redshift, and luminosity-distance dependence. Note that black-hole binaries with masses $M>10^{7} M_{\odot}$ may not coalesce within a Hubble time (Milosavljevic \& Merritt 2003).

of the inspiral signals with analytic approximations to the mergers. Already such a model has been proposed, which provides high overlaps with the numerical simulations for massratios as low as $q=1 / 4$ (Buonanno et al. $\mid 2007 b)$.

Applying the mass scalings from Flanagan \& Hughes (1998), we can estimate the effect of the mass ratio $q$ on the computed SNRs; specifically, SNR $\sim \eta^{1 / 2}$ for the inspiral, and SNR $\sim \eta$ for the merger and ringdown, where $\eta \equiv m_{1} m_{2} / M^{2}=q /(1+q)^{2}$ is the symmetric mass ratio. For stellar-mass black-hole binaries, the mass ratios are rather broadly distributed (Belczynski et al. 2007); the rates for such mergers may be low, $2 y r^{-1}$ for adLIGO, depending on the evolution of the original binary through the common envelope phase. For intermediate-mass black-hole binaries, mass ratios in the range $0.1 \lesssim$ $q \lesssim 1$ are expected to be the most relevant, with potential rates of $\sim 10$ per year (Fregeau et al. 2006); we note, however, that these rates are far more uncertain than those for stellar-mass black-hole binaries. Investigations of gravitational-wave data analysis based on unequal-mass numerical simulations are now underway: Pan et al. (2007) have investigated how numerical relativity waveforms may be used to select the best postNewtonian template banks for searches in the LIGO, adLIGO and VIRGO data stream.

Astrophysical black-hole binaries are also expected to be spinning, which can potentially raise the SNR, for example if there is a spin-induced hangup that generates more gravitational wave cycles in the merger (Campanelli et al. 2006d). Vaishnav et al. (2007) have investigated how the addition of spins to equal-mass waveforms affects search templates, necessitating the use of higher-order modes for high matches in the LIGO band.

We also note that, with more accurate computational modeling, simulations of the merger can be used together with gravitational wave observations to probe gravity in the regime of strong fields. In particular, if the binary masses and spins can be obtained with 


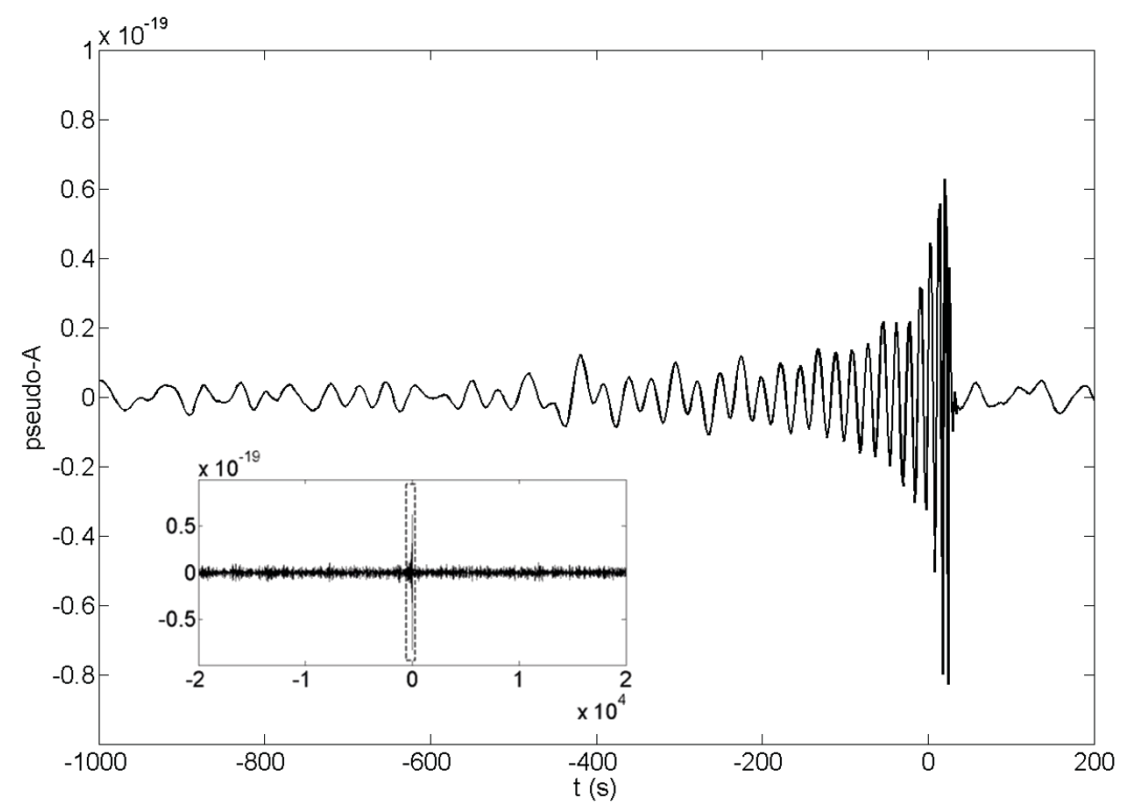

Figure 4 Simulated LISA data stream showing LISA's response to a system of two equalmass black holes. Specifically, the pseudo-A observable is shown for the case of two $4 \times 10^{5} M_{\odot}$ black holes at redshift $z=10$, inclination $\iota=\pi / 2$, polarization angle $\psi=0$, ecliptic latitude $\beta=0$, and ecliptic longitude $\lambda=\pi$. The pseudo-A, E, and T observables are a set of linearly independent combinations of the Michelson unequal arm X, Y, and Z observables at TDI 1.5 (Shaddock et al. 2003), and are therefore useful in data analysis. The inset shows a time span of $4 \times 10^{4}$ seconds encompassing the time span of the main figure, the main figure being an enlargement of the dash-boxed region of the inset.

good accuracy from observations of the inspiral with LISA (Lang \& Hughes 2006), the merger waveform can be calculated using numerical relativity. With this, a comparison can be made between the predictions of general relativity - or any other theory of gravity used in a numerical simulation - with observations in the regime of very strong gravity.

\section{Kicks from black-hole binary mergers}

Black-hole binaries that possess an asymmetry, such as having unequal masses or possessing unequal or unaligned spins, will emit gravitational radiation asymmetrically. This asymmetric gravitational radiation will impart a linear momentum recoil on the binary's center of mass. Previous attempts to calculate this recoil, or "kick", analytically in the nonspinning case (Fitchett 1983) and for cases with spin (Kidder 1995) included very large uncertainties, because the dominant part of the kick occurred in the last orbit through the merger, which was very poorly modeled. With the advent of stable, accurate numerical relativity codes, the problem of accurately calculating the kick became possible. Several groups have tackled this problem. The problem of kicks from an unequal mass binary was first investigated in Baker et al. $(2006 d)$ ), where recoils between 86 and $115 \mathrm{~km} / \mathrm{s}$ for a $q=2 / 3$ mass ratio were simulated. In Gonzalez et al. (2007b), several 
simulations were performed and a peak recoil velocity for nonspinning binaries of 175 $\mathrm{km} / \mathrm{s}$ was found for a mass ratio of $q \approx 1 / 2.78$.

A flurry of activity began when several groups began simulating kicks from spin asymmetry. Herrmann et al. (2007) demonstrated that kicks from spin asymmetry can exceed unequal-mass kicks, and that for an equal-mass configuration with one black hole spinning prograde and the other spinning retrograde with equal magnitude $\hat{a}$ (a dimensionless spin parameter, varying from 0 for a Schwarzschild hole to 1 for a maximally spinning Kerr hole), the spin kick scales as $475 \hat{a} \mathrm{~km} / \mathrm{s}$. However, it was soon found that the largest kicks are obtained when the spins are antiparallel with each other but perpendicular to the orbital angular momentum, a configuration suggested by Campanelli et al. $(2007 a)$. Gonzalez et al. $(2007 a)$ were the first to perform this simulation, obtaining a kick of $2650 \mathrm{~km} / \mathrm{s}$ for $\hat{a}=0.8$. Campanelli et al. $(2007 b)$ showed that the resulting kick depends sensitively on the angle between the black-hole spin vectors and their velocities. They found a simple cosine dependence, indicating that Gonzalez et al. $(2007 a)$ somewhat fortuitously happened upon what is nearly the maximum angle. Furthermore, Campanelli et al. (2007b) projected that for maximal spin, kicks as large as $4000 \mathrm{~km} / \mathrm{s}$ are possible.

Kicks of $2000 \mathrm{~km} / \mathrm{s}$ are sufficiently large to kick remnant black holes out of even the largest giant elliptical galaxies. Therefore, simulation results this large had to be reconciled with the observational reality that we see massive black holes at the centers of all the galaxies that we have observed above a certain spheroidal mass threshold. Schnittman \& Buonanno (2007) used the EOB formalism to perform a Monte Carlo simulation in order to try and predict how typical these large kicks are. For mass ratios between $q=1$ and $q=1 / 10$ and spins of $\hat{a}=0.9$ on both holes, they allowed random orientations among the spin vectors and the orbital angular momentum. They found that $12 \%$ of the remnants received a kick exceeding $500 \mathrm{~km} / \mathrm{s}$, and $2.7 \%$ received a kick exceeding $1000 \mathrm{~km} / \mathrm{s}$.

Schnittman \& Buonanno (2007) assume no external influence which might tend to align the spins with each other and with the orbital angular momentum. However, Bogdanovic, Revnolds \& Miller (2007) investigated the role of the Bardeen-Petterson effect (Bardeen \& Petterson 1975) in mitigating the gravitational recoil from spin asymmetry. In the absence of gas accretion, the assumption of random orientation in Schnittman \& Buonanno (2007) applies. However, if a black-hole binary accretes (1-10)\% of its initial mass from an accretion disk, the spin of each hole will align with the angular momentum of the disk, which in turn is aligned with the orbital angular momentum of the binary. Therefore, if the majority of mergers occur in gas-rich environments, then the giant kick results, although theoretically interesting, are not astrophysically relevant. There is good reason to expect supermassive black holes to accrete substantial mass, particularly at larger redshift. In this scenario, the configuration of greatest interest is spin orientations aligned with the orbital angular momentum.

Fig. [5]and Table 1 present the results of our investigation of this class of configurations, which were first presented in Baker et al. (2007a). "NE" refers to unequal mass, with the corresponding simulations having a mass ratio $q=2 / 3$. "+" means prograde with respect to the orbital angular momentum, and "-" means retrograde ("0" means no spin). Fig. 5 shows the accumulated kick as a function of time for all of our runs. Of particular note is the absence of an "unkick" for the equal-mass case, and the variable size of the unkick in the other cases. Also, we observe the expected accelerated merger in the $\mathrm{NE}_{--}$and $\mathrm{NE}_{0-}$ cases due to spin-orbit attraction, and correspondingly the delayed merger in the $\mathrm{NE}_{++}$and $\mathrm{NE}_{0+}$ cases due to spin-orbit repulsion. Using our data along with the data from Herrmann et al. (2007) and Koppitz et al. (2007) (which are also included in Table 
Table 1. Predicted versus computed kick speed. Runs labeled "S0.\#\#" are taken from Herrmann et al. (2007), while runs labeled "r\#" are taken from Koppitz et al. (2007). [from Baker et al. (2007a) - reproduced with permission of the AAS]

\begin{tabular}{|c|c|c|c|c|c|c|}
\hline Run & $q$ & $\hat{a}_{1}$ & $\hat{a}_{2}$ & $v_{\text {num }}$ & $v_{\text {pred }}$ & $\frac{|\Delta v|}{v_{\text {num }}}(\%)$ \\
\hline $\mathrm{NE}_{--}$ & 0.654 & -0.201 & -0.194 & 116.3 & 119.5 & 2.7 \\
\hline $\mathrm{NE}_{-+}$ & 0.653 & -0.201 & 0.193 & 58.5 & 58.2 & 0.5 \\
\hline $\mathrm{NE}_{0-}$ & 0.645 & 0.000 & -0.195 & 167.7 & 153.1 & 8.7 \\
\hline $\mathrm{NE}_{00}$ & 0.677 & 0.000 & 0.000 & 95.8 & 98.6 & 2.9 \\
\hline $\mathrm{NE}_{0+}$ & 0.645 & 0.000 & 0.194 & 76.9 & 71.7 & 6.8 \\
\hline $\mathrm{NE}_{+-}$ & 0.655 & 0.201 & -0.194 & 188.6 & 181.9 & 3.6 \\
\hline $\mathrm{NE}_{++}$ & 0.654 & 0.201 & 0.194 & 83.4 & 92.4 & 10.8 \\
\hline $\mathrm{EQ}_{+-}$ & 1.001 & 0.198 & -0.198 & 89.8 & 92.6 & 3.2 \\
\hline S0.05 & 1.000 & 0.200 & -0.200 & 96.0 & 93.8 & 2.3 \\
\hline S0.10 & 1.000 & 0.400 & -0.400 & 190.0 & 187.6 & 1.2 \\
\hline S0.15 & 1.000 & 0.600 & -0.600 & 285.0 & 281.5 & 1.2 \\
\hline S0.20 & 1.000 & 0.800 & -0.800 & 392.0 & 375.3 & 4.3 \\
\hline r0 & 1.000 & -0.584 & 0.584 & 260.0 & 274.0 & 5.4 \\
\hline r1 & 0.917 & -0.438 & 0.584 & 220.0 & 220.8 & 0.3 \\
\hline r2 & 0.872 & -0.292 & 0.584 & 190.0 & 178.1 & 6.3 \\
\hline r3 & 0.848 & -0.146 & 0.584 & 140.0 & 141.9 & 1.4 \\
\hline r4 & 0.841 & 0.000 & 0.584 & 105.0 & 110.4 & 5.1 \\
\hline
\end{tabular}

1), we are able to construct an empirical kick formula, given by:

$$
v=V_{0}\left[32 q^{2} /(1+q)^{5}\right] \sqrt{(1-q)^{2}+2(1-q) K \cos \theta+K^{2}},
$$

where $K=k\left(q \hat{a}_{1}-\hat{a}_{2}\right)$. The parameter $V_{0}$ gives the overall scaling of the kick (note that the factor in brackets becomes unity for $q=1$ ), while $k$ gives the relative scaling of the kick contributions from spin and mass asymmetries.

Performing a least-squares fit to the data yields $V_{0}=276 \mathrm{~km} / \mathrm{s}, q=0.58$, and $k=0.85$. Our formula yields a maximum error of $10.8 \%$ for the cases investigated. If, in fact, the majority of supermassive black-hole mergers are gas-rich, then this formula may predict a significant component of the kick for the majority of cases of astrophysical interest. Campanelli et al. $(2007 \mathrm{~b})$ subsequently suggested an extension of the formula presented in Baker et al. (2007a) for kicks out of the orbital plane, which would be more applicable in gas-poor cases where the Bardeen-Petterson effect doesn't occur, such as gas-poor galaxies and the merger of stellar-mass black holes. The achievement of simulating the merger is therefore leading us to a fairly comprehensive understanding of the recoil velocity parameter space in a strikingly brief period of time.

\section{Summary}

Mergers of comparable-mass black-hole binaries produce intense bursts of gravitational radiation and are very strong sources for both ground-based interferometers and the space-based LISA. Recent progress in numerical relativity now makes it possible to 


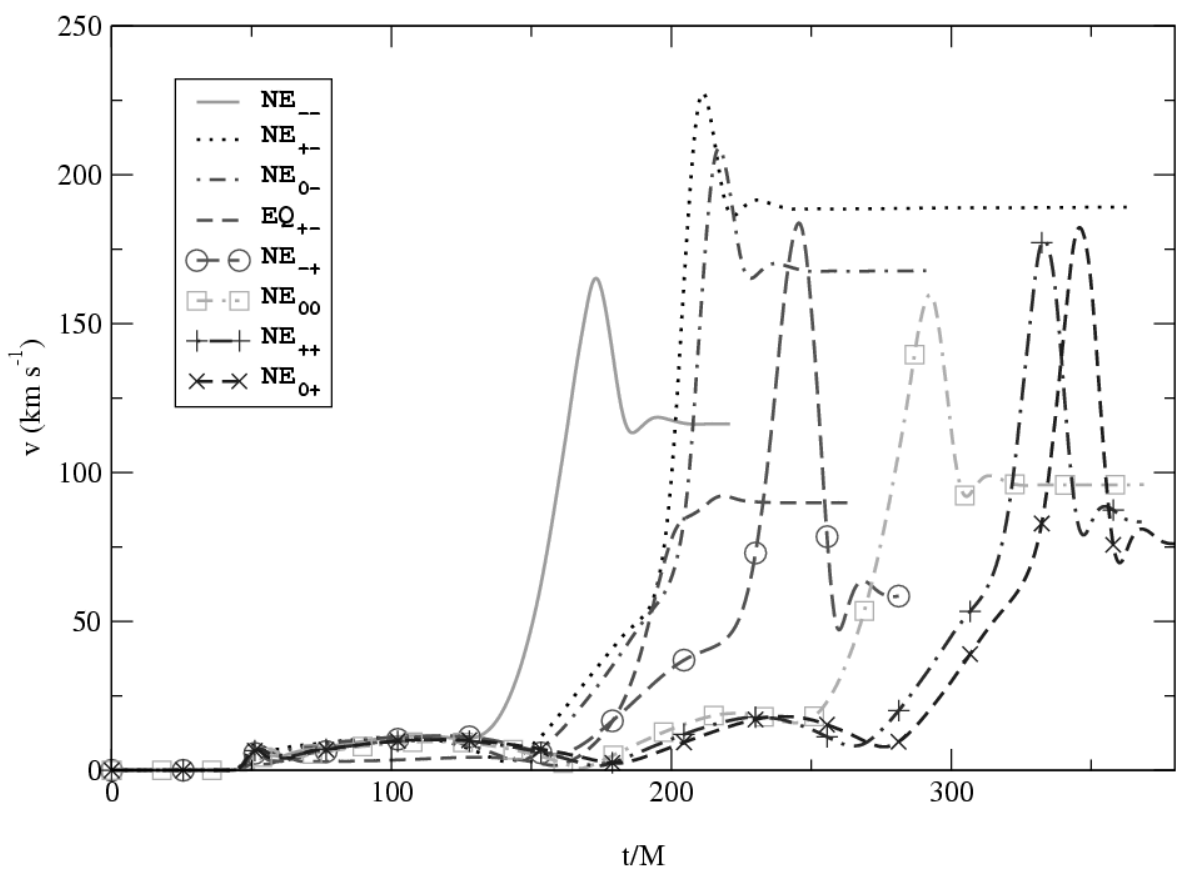

Figure 5 Aggregated kicks from all of our runs. The merger time for each binary matches the peak in its kick profile; the relative delay in merger times between data sets differing in initial spins is consistent with the results of Campanelli et al. (2006d). All configurations show a marked "unkick" after the peak, with the exception of the equal-mass case, $\mathrm{EQ}_{+-}$. [from Baker et al. (2007a) - reproduced with permission of the AAS]

calculate the merger waveforms accurately and robustly. Today there is consensus that the merger of equal-mass, nonspinning black holes produces a final Kerr black hole with spin $a / m_{f} \sim 0.7$, and that the amount of energy radiated in the form of gravitational waves, starting with the final few orbits and proceeding through the plunge, merger and ringdown, is $E_{\mathrm{rad}}=M-m_{f} \sim 0.04 M$. Simulations are now being carried out for an increasing range of black-hole component masses and spins. Computational methods continue to improve, resulting in longer simulations that start in the late inspiral regime and allow quantitative comparisons with analytic post-Newtonian methods. Applications of the resulting waveforms to gravitational wave data analysis have begun. The recoil or kick velocity that results when the black holes have unequal masses, or unequal or unaligned spins, has been calculated for a variety of interesting cases, with key applications to astrophysics. Overall, the field of black-hole binary mergers is experiencing a true "golden age," with many new results coming out across a broad front. Stay tuned!

It is a pleasure to thank our colleagues and collaborators Alessandra Buonanno, Scott Hughes, Cole Miller, Jeremy Schnittman and Tuck Stebbins for stimulating discussions. This work was supported in part by NASA grant 06-BEFS06-19. The simulations were carried out using Project Columbia at the NASA Advanced Supercomputing Division (Ames Research Center), and at the NASA Center for Computational Sciences (Goddard Space Flight Center). B.J.K. was supported by the NASA Postdoctoral Program at the Oak Ridge Associated Universities. S.T.M. was supported in part by the Leon A. Herreid Graduate Fellowship. 


\section{REFERENCES}

Аввотт, B. et al. 2007 Search for gravitational-wave bursts in LIGO data from the fourth science run. arXiv:0704.0943 [gr-qc].

Alcubierre, M., Benger, W., Brügmann, B., Lanfermann, G., Nerger, L., Seidel, E. \& Takahashi, R. 2001 3D Grazing Collision of Two Black Holes. Phys. Rev. Lett. 87, 271103.

Arnowitt, R., Deser, S. \& Misner, C. W. 1962 The dynamics of general relativity. In Gravitation: An Introduction to Current Research (ed. L. Witten), pp. 227-265. New York: John Wiley.

Baker, J., Campanelli, M., Pretorius, F. \& Zlochower, Y. $2006 a$ Comparisons of binary black hole merger waveforms. Class. Quantum Grav. 24, S25-S31.

Baker, J. G., Boggs, W. D., Centrella, J., Kelly, B. J., McWilliams, S. T., Miller, M. C. \& van Meter, J. R. 2007 a Modeling kicks from the merger of non-precessing black-hole binaries. Astrophys. J. In press.

Baker, J. G., Centrella, J., Choi, D.-I., Koppitz, M. \& van Meter, J. $2006 b$ Binary black hole merger dynamics and waveforms. Phys. Rev. D 73, 104002.

Baker, J. G., Centrella, J., Choi, D.-I., Koppitz, M. \& van Meter, J. 2006c Gravitational wave extraction from an inspiraling configuration of merging black holes. Phys. Rev. Lett. 96, 111102.

Baker, J. G., Centrella, J., Choi, D.-I., Koppitz, M., van Meter, J. \& Miller, M. C. $2006 d$ Getting a kick out of numerical relativity. Astrophys. J. Lett. 653, L93.

Baker, J. G., McWilliams, S. T., van Meter, J. R., Centrella, J., Choi, D.-I., Kelly, B. J. \& Koppitz, M. $2007 b$ Binary black hole late inspiral: Simulations for gravitational wave observations. Phys. Rev. D 75, 124024.

Baker, J. G., van Meter, J. R., McWilliams, S. T., Centrella, J. \& Kelly, B. J. $2006 e$ Consistency of post-Newtonian waveforms with numerical relativity. gr-qc/0612024

Bardeen, J. M. \& Petterson, J. A. 1975 The Lense-Thirring effect and accretion disks around Kerr black holes. Astrophys. J. 195, L65-L67.

Belczynski, K., Kalogera, V., Rasio, F. A., TaAm, R. E. \& Bulik, T. 2007 On the rarity of double black hole binaries: Consequences for gravitational wave detection. Astrophys. J. 662, 504-511.

Blanchet, L. 2006 Gravitational radiation from post-Newtonian sources and inspiralling compact binaries. Living Rev. Rel. 9, 4.

Bogdanovic, T., Reynolds, C. S. \& Miller, M. C. 2007 Alignment of the spins of supermassive black holes prior to coalescence. Astrophys. J. 661, L147-L150.

Brandt, S., Correll, R., Gómez, R., Huq, M. F., Laguna, P., Lehner, L., Marronetti, P., Matzner, R. A., Neilsen, D., Pullin, J., Schnetter, E., Shoemaker, D. \& Winicour, J. 2000 Grazing collisions of black holes via the excision of singularities. Phys. Rev. Lett. 85, 5496-5499.

Brügmann, B. 1999 Binary black hole mergers in 3D numerical relativity. Int. J. Mod. Phys. D 8, 85 .

Brügmann, B., Tichy, W. \& Jansen, N. 2004 Numerical simulation of orbiting black holes. Phys. Rev. Lett. 92, 211101.

Buonanno, A., Cook, G. B. \& Pretorius, F. $2007 a$ Inspiral, merger and ring-down of equal-mass black-hole binaries. Phys. Rev. D 75, 124018.

Buonanno, A., Pan, Y., Baker, J. G., Centrella, J., Kelly, B. J., McWilliams, S. T. \& VAn Meter, J. R. $2007 b$ Toward faithful templates for non-spinning binary black holes using the effective-one-body approach. arXiv:0706.3732 [gr-qc].

Campanelli, M., Lousto, C. O., Marronetti, P. \& Zlochower, Y. 2006 a Accurate evolutions of orbiting black-hole binaries without excision. Phys. Rev. Lett. 96, 111101.

Campanelli, M., Lousto, C. O. \& Zlochower, Y. $2006 b$ Last orbit of binary black holes. Phys. Rev. D 73, 061501(R).

Campanelli, M., Lousto, C. O. \& Zlochower, Y. 2006 c Spin-orbit interactions in black-hole binaries. Phys. Rev. D 74, 084023.

Campanelli, M., Lousto, C. O. \& Zlochower, Y. 2006d Spinning-black-hole binaries: The orbital hang up. Phys. Rev. D 74, 041501(R). 
Campanelli, M., Lousto, C. O., Zlochower, Y. \& Merritt, D. 2007 a Large merger recoils and spin flips from generic black-hole binaries. Astrophys. J. 659, L5-L8.

Campanelli, M., Lousto, C. O., Zlochower, Y. \& Merritt, D. $2007 b$ Maximum gravitational recoil. Phys. Rev. Lett. 98, 231102.

Centrella, J. M. 2006 The final merger of comparable mass binary black holes. AIP Conf. Proc. 873, 70-79.

ECheverría, F. 1989 Gravitational-wave measurements of mass and angular momentum of a black hole. Phys. Rev. D 40, 3194.

Fitchetт, M. J. 1983 The influence of gravitational wave momentum losses on the centre of mass motion of a Newtonian binary system. Mon. Not. R. Astron. Soc. 203, 1049-1062.

Flanagan, E. E. \& Hughes, S. A. 1998 Measuring gravitational waves from binary black hole coalescence: I. signal to noise for inspiral, merger, and ringdown. Phys. Rev. D 57, 4535.

Fregeau, J. M., Larson, S. L., Miller, M. C., O’Shaughnessy, R. \& Rasio, F. A. 2006 Observing IMBH-IMBH binary coalescences via gravitational radiation. Astrophys. J. 646, L135.

Gonzalez, J. A., Hannam, M. D., Sperhake, U., Brügmann, B. \& Husa, S. 2007 a Supermassive recoil velocities for binary black-hole mergers with antialigned spins. Phys. Rev. Lett. 98, 231101.

Gonzalez, J. A., Sperhake, U., Brügmann, B., Hannam, M. \& Husa, S. $2007 b$ Maximum kick from nonspinning black-hole binary inspiral. Phys. Rev. Lett. 98, 091101.

Hahn, S. G. \& Lindquist, R. W. 1964 The two body problem in geometrodynamics. Ann. Phys. 29, 304-331.

Hannam, M., Husa, S., Sperhake, U., Brügmann, B. \& Gonzalez, J. A. 2007 Where post-Newtonian and numerical-relativity waveforms meet. arXiv:0706.1305 [gr-qc].

Herrmann, F., Hinder, I., Shoemaker, D., Laguna, P. \& Matzner, R. A. 2007 Gravitational recoil from spinning binary black hole mergers. gr-qc/0701143

Herrmann, F., Shoemaker, D. \& Laguna, P. 2006 Unequal-mass binary black hole inspirals

Husa, S., Gonzalez, J. A., Hannam, M., Brügmann, B. \& Sperhake, U. $2007 a$ Reducing phase error in long numerical binary black hole evolutions with sixth order finite differencing. arXiv:0706.0740 [gr-qc].

Husa, S., Hannam, M., Gonzalez, J. A., Sperhake, U. \& Brügmann, B. $2007 b$ Reducing eccentricity in black-hole binary evolutions with initial parameters from post-Newtonian inspiral. arXiv:0706.0904 [gr-qc].

KIDDER, L. E. 1995 Coalescing binary systems of compact objects to (post) ${ }^{5 / 2}$-Newtonian order. V. Spin effects. Phys. Rev. D 52, 821-847.

Koppitz, M., Pollney, D., Reisswig, C., Rezzolla, L., Thornburg, J., Diener, P. \& Schnetter, E. 2007 Recoil velocities from equal-mass binary-black-hole mergers. Phys. Rev. Lett. 99, 041102.

LAng, R. N. \& Hughes, S. A. 2006 Measuring coalescing massive binary black holes with gravitational waves: The impact of spin-induced precession. Phys. Rev. D 74, 122001.

LEAVER, E. W. 1986 An analytic representation for the quasi-normal modes of Kerr black holes. Proc. R. Soc. London Ser. A 402 (1823).

van Meter, J. R., Baker, J. G., Koppitz, M. \& Choi, D.-I. 2006 How to move a black hole without excision: gauge conditions for the numerical evolution of a moving puncture. Phys. Rev. D 73, 124011.

Milosavljevic, M. \& MerRitt, D. 2003 Long term evolution of massive black hole binaries. Astrophys. J. 596, 860 .

Misner, C. W., Thorne, K. S. \& Wheeler, J. A. 1973 Gravitation. San Francisco: W. H. Freeman.

Pan, Y., Buonanno, A., Baker, J. G., Centrella, J., Kelly, B. J., McWilliams, S. T., Pretorius, F. \& van Meter, J. R. 2007 A data-analysis driven comparison of analytic and numerical coalescing binary waveforms: non-spinning case. Submitted to Phys. Rev. D.

Pfeiffer, H. P. et al. 2007 Reducing orbital eccentricity in binary black hole simulations. Class. Quantum Grav. 24, S59-S82.

Pretorius, F. 2005 Evolution of binary black hole spacetimes. Phys. Rev. Lett. 95, 121101. 
Schnittman, J. D. \& Buonanno, A. 2007 The distribution of recoil velocities from merging black holes. astro-ph/0702641.

Sesana, A., HaArdt, F., Madau, P. \& Volonteri, M. 2005 The contribution of inspiraling massive black hole binaries to the LISA data stream. Astrophys. J. 623, 23-30.

Shaddock, D. A., Tinto, M., Estabrook, F. B. \& Armstrong, J. W. 2003 Data combinations accounting for LISA spacecraft motion. Phys. Rev. D 68, 061303.

Shoemaker, D. 2006 Private communication.

SMARR, L. 1977 Spacetimes generated by computers: Black holes with gravitational radiation. Ann. N. Y. Acad. Sci. 302, 569-604.

SmARR, L. 1979 Gauge conditions, radiation formulae and the two black hole collision. In Sources of Gravitational Radiation (ed. L. Smarr), p. 245. Cambridge, England: Cambridge University Press.

Smarr, L., Čadež, A., DeWitt, B. \& Eppley, K. 1976 Collision of two black holes: Theoretical framework. Phys. Rev. D 14 (10), 2443-2452.

Tichy, W. \& Marronetti, P. 2007 Binary black hole mergers: Large kicks for generic spin orientations. gr-qc/0703075.

Vaishnav, B., Hinder, I., Herrmann, F. \& Shoemaker, D. 2007 Matched filtering of numerical relativity templates of spinning binary black holes. arXiv:0705.3829 [gr-qc]. 